\title{
EFEITO DOS NÍVEIS DE CÁLCIO DA RAÇÃO SUPLEMENTADA COM FITASE SOBRE A DEPOSIÇÃO DE MINERAIS NA TÍBIA DE FRANGOS DE CORTE DE 22 A 42 DIAS
}

\author{
NEUDI ARTEMIO SCHOULTEN ${ }^{1}$ \\ ANTÔNIO SOARES TEIXEIRA ${ }^{2}$ \\ ADEMIR JOSÉ CONTE ${ }^{3}$ \\ HUNALDO OLIVEIRA SILVA ${ }^{4}$ \\ ANTÔNIO GILBERTO BERTECHINI ${ }^{5}$ \\ ELIAS TADEU FIALHO ${ }^{2}$
}

\begin{abstract}
RESUMO - Com o objetivo de avaliar os efeitos dos níveis de cálcio na ração suplementada com fitase, para frangos de corte de 22 a 42 dias de idade, sobre a deposição de minerais nas tíbias, foi conduzido este experimento, com 600 frangos de 22 dias de idade da linhagem Hubbard-MPK, distribuídos em um delineamento inteiramente casualizado, com arranjo fatorial 5 x 2 (níveis de cálcio da ração x sexos), três repetições de 20 aves por parcela. A ração basal foi balanceada, suplementada com fitase para atender às exigências nutricionais das aves, conforme o NRC (1994), exceto para o
\end{abstract}

cálcio, cujos níveis foram 0,$40 ; 0,59 ; 0,78 ; 0,97$ e $1,16 \%$. Aos 42 dias de idade, duas aves de cada tratamento foram abatidas para retirada da tíbia esquerda para analises posteriores. Os teores de cinzas nas tíbias das fêmeas foram 3,5\% superior aos dos machos. A deposição de cinzas, cálcio, fósforo, zinco, manganês e magnésio não foi afetada $(\mathrm{P}>0,05)$ pelos níveis de cálcio na ração de 22 a 42 dias de idade. Conclui-se que o aumento dos teores de cálcio da ração suplementada com fitase não afeta a deposição de minerais nas tíbias dos frangos dos 22 aos 42 dias de idade.

TERMOS PARA INDEXAÇÃO: Cinza, fósforo, manganês, mineralização, zinco.

\section{EFFECT OF THE LEVELS OF CALCIUM IN DIETS SUPPLEMENTED WITH PHYTASE ON THE DEPOSITION OF MINERALS IN THE TIBIA OF BROILER FROM 22 TO 42 DAYS}

\begin{abstract}
With the aim of evaluating the effects of the levels of calcium in the ration supplemented with phytase for broiler chickens from 22 to 42 days old, on the deposition of minerals in the tibias, one experiment was conducted with 600 chickens of 22 days old (Hubbard-MPK), distributed in a completely randomized design, in $5 \times 2$ factorial arrangement (levels of $\mathrm{Ca}$ of the diet $\mathrm{x}$ sexes), with three repetitions of 20 birds per plot. The basal ration was balanced to meet the nutritional requirements of the birds according to NRC (1994), except for Ca, whose levels were 0.40;
\end{abstract}

$0.59 ; 0.78 ; 0.97$ and $1.16 \%$, supplemented with phytase . At 42 days of age, two chickens from each treatment were slaughtered for removal of the left tibia for the subsequent analyses. The contents of ashes in the tibia from female were $35 \%$ higher than those of the males. The deposition of ashes, $\mathrm{Ca}, \mathrm{P}, \mathrm{Zn}, \mathrm{Mn}$ and $\mathrm{Mg}$ were not affected $(\mathrm{P}>0.05)$ by the levels of calcium in the ration supplemented with phytase. In conclusion the increasing levels of calcium with phytase supplementation in the diets did not affect the deposition of minerals in the tibias of chickens from 22 to 42 days old.

INDEX TERMS: Ash, manganese, mineralization, phosphorus, zinc.

1. Lic. Cienc. Agr., M.Sc., Professor da Escola Agrotécnica. Federal de Rio do Sul, SC. neudi@navinet,com.br

2 Engenheiro Agrônomo, D.Sc., Professor do Departamento de Zootecnia da UNIVERSIDADE FEDERAL DE LAVRAS/UFLA. Caixa Postal 37, 37200-000 - Lavras, MG,

3. Lic. Cienc. Agr., D.Sc., Professor da Escola Agrotécnica Federal de Cuiabá, MT. ajconte@zaz,com.br

4. Médico Veterinário, M.Sc., Estudante de Doutorado DZO/UFLA. hunaldo@ufla.br

5. Zootecnista, D.Sc., Professor do Departamento de Zootecnia/UFLA. 


\section{INTRODUÇÃO}

A adequada mineralização óssea é de fundamental importância na avicultura de corte, já que o desenvolvimento muscular é dependente de um bom suporte ósseo e é necessário para o bom funcionamento do sistema locomotor. Aves com deficiências no desenvolvimento ósseo podem sofrer fraturas durante a operação de apanha, transporte e abate, acarretando grandes prejuízos em virtude de condenações de carcaças no abatedouro. No que se refere às necessidades de minerais para aves, os macrominerais, como cálcio e fósforo, são os de maior importância. $\mathrm{O}$ cálcio tem sido alvo de vários trabalhos de pesquisa, nos quais se procura determinar a exigência nutricional desse elemento, considerando as diferentes linhagens, sexo, consumo de ração e suas relações com outros minerais da ração, principalmente o fósforo. Conforme o NRC (1994), a exigência de cálcio para frangos é de $1 \%$ de 1 a 21 dias e $0,90 \%$ de 22 a 42 dias, para rações com $3200 \mathrm{kcal} / \mathrm{kg}$. Porém, Karunajeewa (1976) concluiu que para a fase de crescimento, $0,60 \%$ de cálcio e $0,57 \%$ de fósforo, respectivamente, são suficientes para a adequada taxa de crescimento e mineralização óssea, quando os minerais estão presentes em formas altamente disponíveis.

Níveis de cálcio e fósforo na ração para frangos suplementadas com fitase afetam a utilização do fósforo fítico (Edwards Junior \& Veltmann, 1983). Aves que consomem rações com baixos níveis de fósforo e cálcio inorgânico possuem maior capacidade para hidrolisar o fitato do que aquelas que recebem níveis altos (Denbow et al., 1995). A relação cálcio/fósforo influencia a atividade da fitase, que é reduzida com a elevação do nível de cálcio da ração (Qian et al., 1997). Em rações práticas suplementadas com fitase, essa relação parece mais crítica do que quantidades individuais desses minerais. A elevação da proporção de cálcio/fósforo reduz significativamente o desempenho de frangos alimentadas com rações à base de milho e farelo de soja, suplementadas com fitase (Leeson 1999), provavelmente por causa da reação do cálcio com o ácido fítico, formando o fitato de cálcio, que precipita e não pode ser atacado pela fitase. Ao centrifugar o conteúdo intestinal de frangos de corte que consumiram rações com alto nível de cálcio (acima de 1,53\%), Shafey et al. (1991) verificaram que $70 \%$ a $92 \%$ do cálcio, ferro, magnésio e zinco estavam na forma insolúvel e sugerem que a elevação do pH intestinal, em função da elevação do nível de cálcio, reduz a fração solúvel de minerais e a sua disponibilidade para absorção; dessa forma, espera-se que as características ósseas também sejam afetadas. Para uma maior solubilização do fitato, é necessário manter os ní- veis de fósforo e cálcio inorgânicos na ração nos limites mínimos necessários (Mitchell \& Edwards Junior, 1996); Com isso, sugere-se que os níveis desses minerais devem ser reduzidos quando a fitase for empregada durante a formulação (Ballam et al., 1984). A mineralização óssea de frangos de corte de 1 a 21 dias de idade, submetidos à ração à base de milho e farelo de soja, com $0,51 \%$ de fósforo total, suplementada com $66 \mu \mathrm{g}$ de vitamina D e 600 FTU/kg de ração, foi afetada pelos níveis de cálcio da ração com resposta quadrática, com redução dos teores de cinza dos ossos à medida que o nível de cálcio foi elevado de 0,56 para 1,02\% (Qian et al., 1997).

Assim, julga-se importante verificar os efeitos dos níveis de cálcio da ração suplementada com fitase sobre a deposição de cinzas, cálcio, fósforo, zinco, manganês e magnésio na tíbia de frangos de corte de 22 a 42 dias de idade.

\section{MATERIAL E MÉTODOS}

$\mathrm{O}$ experimento foi realizado utilizando um total de 600 frangos da marca comercial Hubbard-MPK, no período de 22 a 42 dias, alojadas em galpão sobre cama, distribuídos em cinco tratamentos e seis repetições, sendo três de cada sexo, e conduzido no Setor de Avicultura da Escola Agrotécnica Federal de Cuiabá (MT), em janeiro e fevereiro de 2000. Os tratamentos foram dispostos em um delineamento inteiramente casualizado, em esquema fatorial 5 × 2 (cinco níveis de cálcio na ração: 0,$40 ; 0,59 ; 0,78 ; 0,97$ e $1,16 \%$, obtidos pela substituição do caulim da ração basal por calcário, e dois sexos). As rações foram isocalóricas, isoprotéicas e com os mesmos teores de vitaminas, minerais e aminoácidos, à base de milho e farelo de soja, com $0,43 \%$ de fósforo total, suplementadas com 600 unidades de fitase $/ \mathrm{kg}$, formuladas para atender às exigências nutricionais, conforme recomendado pelo NRC (1994), exceto para o cálcio, sendo a composição dos ingredientes obtida de Rostagno et al. (1994). Na fase pré-experimental, as aves foram alimentadas com uma ração balanceada para atender às exigências nutricionais, conforme o NRC (1994). A disponibilidade do fósforo de origem vegetal foi considerada como sendo de dois terços, tendo em vista a suplementação com fitase. Foi usada uma enzima comercial, obtida de fungos (Aspergillus niger), com atividade mínima inicial declarada pelo fornecedor de 5000 unidades de fitase (FTU) por grama. A composição da ração basal pode ser observada na Tabela 1. Ração e água foram fornecidas à vontade e mantido um programa de 24 horas de luz (natural + artificial) durante todo o período experimental, utilizando lâmpadas de 
60 watts. Para a retirada da tíbia, foram escolhidas aleatoriamente duas aves na média de peso de cada parcela experimental, totalizando 10 aves por tratamento e um total de 60 aves. Após abate das aves por deslocamento cervical, as tíbias foram retiradas, identificadas e submetidas a tratamento, conforme metodologia descrita por Teixeira (1994). Ingredientes, rações e ossos foram analisados no Laboratório de Pesquisa Animal do DZO/UFLA, segundo metodologia da AOAC (1990).

As análises estatísticas dos resultados obtidos foram realizadas utilizando o programa SISVAR 4.1 (Sistema para Análises de variância de dados balanceados), desenvolvido por Ferreira (2000). A seguir, é descrito o modelo estatístico utilizado:

$$
\mathbf{Y}_{\mathrm{ijk}}=\mu+\mathbf{N}_{\mathrm{i}}+\mathbf{S}_{\mathbf{j}}+\mathbf{N S}_{\mathrm{ij}}+\mathbf{e}_{\mathrm{ijk}}
$$

$\mathrm{Y}_{\mathrm{ijk}}$ - valor observado na unidade experimental k, do nível de cálcio i, do sexo j;

$\mu$ - uma constante associada a todas as observações;

$\mathrm{N}_{\mathrm{i}}$ - o efeito do nível de cálcio, com $\mathrm{i}=1,2,3,4$ e 5;

$\mathrm{S}_{\mathrm{j}}$ - o efeito do sexo, com $\mathrm{j}=1$ e 2 ;

$\mathrm{NS}_{\mathrm{ij}}$ - o efeito da interação do nível de cálcio e do sexo;

$\mathrm{e}_{\mathrm{ijk}}$ - erro experimental associado a $\mathrm{Y}_{\mathrm{ijk}}$ que, por pressuposição, tem distribuição normal com média zero e variância $\sigma^{2}$.

\section{RESULTADOS E DISCUSSÃO}

Os valores médios do conteúdo de cinzas, cálcio, fósforo, zinco, manganês e magnésio na tíbia das aves aos 42 dias de idade são apresentados na Tabela 2.

Em nenhuma variável avaliada houve interação entre os níveis de cálcio e sexos; da mesma forma, não houve diferença entre os sexos e os níveis de cálcio para as variáveis estudadas $(\mathrm{P}>0,05)$. As fêmeas apresentaram deposição de cinzas $(3,54 \%)$ superior $(\mathrm{P}<0,01)$ à dos machos, o que difere dos resultados obtidos por Cabral (1999), que não encontrou diferença entre os sexos na mineralização óssea das tíbias. O maior teor de cinzas das tíbias das fêmeas provavelmente pode ser atribuído à maturidade sexual mais precoce das fêmeas, devido à ação dos hormônios estrogênicos, que propiciam melhor aproveitamento do cálcio, levando a uma calcificação mais intensa (Teixeira 1994). Nelson et al. (1990) e Cabral (1999) encontraram resultados que diferem dos obtidos neste experimento para a deposição de cinzas na tíbia. Para a deposição de cálcio na tíbia, os resultados deste trabalho são semelhantes aos obtidos por Edwards Jr. e Veltmann (1983) e Cabral (1999); no entanto, diferem dos resultados obtidos por Hulan et al. (1985), que verificaram aumento na deposição de cálcio na tíbia com o aumento do nível de cálcio na ração e maior deposição de cálcio na tíbia das fêmeas.

TABELA 1 - Composição da ração basal.

\begin{tabular}{lc}
\hline \multicolumn{1}{c}{ Ingrediente } & Quantidade (kg) \\
\hline Milho & 641,78 \\
Farelo de soja & 296,90 \\
Óleo de soja & 3,90 \\
Sal & 14,78 \\
Premix mineral & 3,00 \\
Premix vitamínico & 0,50 \\
Monoamônio fosfato & 4,00 \\
Fitase & 0,12 \\
Calcário calcítico & 7,52 \\
Caulim & 27,50 \\
\hline Composição calculada & \\
\hline Energia metabolizável (Kcal /kg) & 3000 \\
Proteína bruta (\%) & 19,00 \\
Cálcio (\%) & 0,40 \\
Fósforo disponível (\%) & 0,32 \\
Metionina (\%) & 0,42 \\
Metionina + Cistina (\%) & 0,62 \\
Lisina (\%) & 1,00 \\
Triptofano (\%) & 0,25 \\
Treonina (\%) & 0,75 \\
\hline Oprenix & \\
\hline
\end{tabular}

O premix forneceu por $\mathrm{kg}$ de ração: vit. A - 7.000 UI; vit. $D_{3}-1,500 \mathrm{UI}$; vit. $\mathrm{E}-12 \mathrm{UI}$; vit. $\mathrm{K}-1,5 \mathrm{mg}$; Vit. $B_{1}-1,6 \mathrm{mg}$; vit. $B_{2} 5 \mathrm{mg}$; vit. $B_{6} 2,6 \mathrm{mg}$; vit. $B_{12}$ $10 \mu \mathrm{g}$; Niacina - 35 mg; Ác. Fólico - 0,7 mg; Ác. Pantotênico - $13 \mathrm{mg}$; Se - 0,3 mg; Colina - $300 \mathrm{mg}$; Metionina - 1,0 g; Agente anticoccidiano - 0,06 g; Promotor de crescimento - 0,04 g; Antioxidante 0,02 g; Mn - 75 mg; Zn - 50 mg; Cu - 8 mg; I - 0,75 mg; Fe -50 mg. 
TABELA 2 - Teor de cinzas, cálcio, fósforo, zinco, manganês e magnésio nas tíbias dos frangos aos 42 dias de idade, na base da matéria seca desengordurada.

\begin{tabular}{ccccccc}
\hline & Cinzas $(\boldsymbol{\%})$ & Ca $(\%)$ & P $(\%)$ & Zn (ppm) & Mn (ppm) & Mg (ppm) \\
\hline Nível cálcio & $\mathrm{ns}$ & $\mathrm{ns}$ & $\mathrm{ns}$ & $\mathrm{ns}$ & $\mathrm{ns}$ & $\mathrm{ns}$ \\
0,40 & 54,8 & 18,8 & 10,4 & 171,4 & 8,0 & 2070,7 \\
0,59 & 55,5 & 19,6 & 10,9 & 171,2 & 7,8 & 2292,4 \\
0,78 & 55,0 & 19,7 & 10,9 & 173,4 & 7,7 & 2235,7 \\
0,97 & 56,3 & 20,1 & 11,3 & 180,4 & 7,9 & 2330,4 \\
1,16 & 55,9 & 19,4 & 10,8 & 174,8 & 7,9 & 2137,9 \\
\hline Fêmeas & $56,5 \mathrm{a}$ & $19,5 \mathrm{a}$ & $11,0 \mathrm{a}$ & $174,2 \mathrm{a}$ & $8,0 \mathrm{a}$ & $2272,8 \mathrm{a}$ \\
Machos & $54,5 \mathrm{~b}$ & $19,5 \mathrm{a}$ & $10,8 \mathrm{a}$ & $174,2 \mathrm{a}$ & $7,8 \mathrm{a}$ & $2154,1 \mathrm{a}$ \\
\hline Média & 55,5 & 19,5 & 10,9 & 174,2 & 7,9 & 2213,4 \\
\hline C. V. (\%) & 1,81 & 5,87 & 6,05 & 6,27 & 5,32 & 15,71 \\
\hline
\end{tabular}

Médias seguidas de letras diferentes na mesma coluna diferem pelo teste $\mathrm{F}(\mathrm{P}<0,05)$

ns - não-significativo (P>0,05)

Os resultados deste experimento, nos quais não foram observados efeitos significativos dos níveis de cálcio da ração, provavelmente foram influenciados pelo nível de cálcio da ração na fase pré-experimental, na qual as aves receberam uma ração com $3000 \mathrm{Kcal} / \mathrm{kg}$ e com $0,90 \%$ de cálcio (NRC, 1994), o que foi suficiente para permitir uma adequada deposição de minerais nos ossos, que possivelmente refletiu na fase experimental.

\section{CONCLUSÃO}

Os níveis de cálcio da ração à base de milho e farelo de soja, para frangos de corte na fase de 22 a 42 dias de idade, suplementadas com fitase, não afetam a mineralização óssea.

\section{REFERÊNCIAS BIBLIOGRÁFICAS}

ASSOCIATION OF OFFICIAL ANALYTICAL CHEMIST - AOAC. Official methods of analysis: agricultural chemicals, contaminants and drugs. 15. ed. Washington, 1990. v. 1, 684 p.

BALLAM, G. C.; ENGSTER, H. M.; SNETZINGER, D. C. Effect of calcium level on the ability of broiler and single comb White leghorn to hydrolyze phytate phosphorus. Poultry Science, Atlanta, v. 63, p. 61, Jan. 1984.
CABRAL, G. H. Níveis de cálcio em rações para frango de corte. 1999.107 p. Tese (Doutorado em Zootecnia) - Universidade Federal de Viçosa, Viçosa.

DENBOW, D. M.; RAVINDRAN, V.; KORNEGAY; Y.I. Z.; HULET, R. M. Improving phosphorus availability in soybean meal for broilers by supplemental phytase. Poultry Science, Champaign, v. 74, n. 11, p. 1831-1842, Nov.1995.

EDWARDS JUNIOR, H. M.; VELTMANN, J. R. The role of calcium and phosphorus in the etiology of tibial dyscondroplasia in young chicks. Journal of Nutrition, Bethesda, v. 113, n. 8, p. 1568-1575, Aug. 1983.

FERREIRA, D. F. Sistema de análise estatística para dados balanceados (SISVAR). Lavras: UFLA/DEX, 2000 .

HUlan, H. W.; DeGRoOTe, G., FONTAINE, G. The effect of different totals and ratios of dietary calcium and phosphorus on the performance and incidence of leg abnormalities of male and female broiler chickens. Poultry Science, Champaign, v. 64, p. 1157-1169, Jun. 1985.

LEESON, S. Enzimas para aves. In: SIMPÓSIO INTERNACIONAL SOBRE NUTRIÇÃO DE AVES, 
1999, Campinas, Anais... Campinas: FACTA, 1999. p. 173-185.

KARUNAJEEWA, H. Effect of some feed additives on the performance of broiler chicks fed diets containing high levels of meat and bone meal. Australian Journal of Experimental Agriculture and Animal Husbandry, Melbourne, v. 16, p. 685-690, 1976.

MITCHELL, R. D.; EDWARDS JUNIOR, H. M. Additive effects of 1,25-dihidroxicholecalciferol and phytase on phytate phosphorus utilization and related parameters in broiler chickens. Poultry Science, Champaign, v. 75, n. 1, p. 111-119, Jan. 1996.

NATIONAL RESEARCH COUNCIL - NRC. Nutrient requirements of poultry. 9. ed. Washington, 1994. $155 \mathrm{p}$.

QIAN, H.; KORNEGAY, E. T.; DENBOW, D. M. Utilization of phytate phosphorus and calcium as influenced by microbial phytase, cholecalciferol, and the calcium: total phosphorus ratio in broiler diets. Poultry Science, Champaign, v. 76, n. 5, p. 37-46, Jan. 1997.

ROSTAGNO, H. S.; SILVA, D. J.; COSTA, P. M. A.; FONSECA, J. B.; SOARES, P. R.; PEREIRA, J. A. A.; SILVA, M. A. Composição de alimentos e exigências nutricionais de aves e suínos (Tabelas Brasileiras). Viçosa: UFV, 1994. 59 p.

SHAFEY, T. M.; McDONALD, M. W.; DINGLE, J. G. Effects of dietary calcium and available phosphorus concentration on digesta $\mathrm{pH}$ and on the availability of calcium, iron, magnesium and zinc from the intestinal contents of meat chickens. British Poultry Science, London, v. 32, n. 1, p. 185-194, Mar. 1991.

TEIXEIRA, A. S. Exigências nutricionais de zinco e sua disponibilidade em sulfatos e óxidos de zinco para pintos de corte. $1994.172 \mathrm{p}$. Tese (Doutorado em Zootecnia) - Universidade Federal do Rio Grande do Sul, Porto Alegre. 